\title{
Skin Scarification Route of Administration
}

National Cancer Institute

\section{Source}

National Cancer Institute. Skin Scarification Route of Administration. NCI Thesaurus.

Code C149897.

Administration of a medicinal product by scratching the skin. 